\title{
The Controller Development of Multi-layer Parking Equipment Based on STM32
}

\author{
Zhang Yongde ${ }^{1}$, Zhang Qinxiang ${ }^{1}$, Jiang Jingang ${ }^{1,}$, Zhao Yanjiang $^{1}$, Qian Qingwen ${ }^{1}$, \\ Tang Dedong ${ }^{1}$ and Tian Daifu ${ }^{2}$ \\ ${ }^{1}$ Intelligent Machine Institute, Harbin University of Science and Technology, China \\ ${ }^{2}$ Shangzhi Tiandi Stereoscopic Parking Equipment Manufacturing Co. Ltd.,China

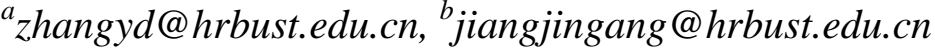

\begin{abstract}
To meet the needs of the general parking equipment with high integration, fully functional control system, we design a controller based on STM32F103 series michoprocessor used in Multi-layer parking equipment, with SD card, NRF and other module interface. To ensure the stability of the controller, all of the input interface relay output interface and the power supply part are isolated, smoothed and other necessary processing; The controller can be performed RS485, CAN bus communication, also with voice alarm, LCD display. After the necessary tests, combined with practical work of the three Cayers and six places parking equipment, then verified the reliability and practicability of the entroller.
\end{abstract}

Keywords: Multi-layer Parking Equipment, STM32, Controller

\section{Introduction}

Automotive and real estate industry are developing rapidly, making the city parking problems become increasingly prominemt Multi-layer Parking Equipment for its safety, good stability and high economic advantages, has been widely used, and its controller mostly uses PLC[1]. However, PL C costs highly, more connected lines, relatively independent of each unit, then can not be a wide range of applications, at the same time can not conducive to management [2]. Therefore, we design a controller used in Multi-layer parking equipment based on STM32F103 series microprocessor, with power supply module, relay module. Support RS485, CAN bos communication, capable of voice prompts, with anti-jamming input interface and rich peripheral interfaces, be able to facilitate future software upgrades and equipment improrements. The controller not only can guarantee real-time control, stability and accuracy, but also can reduce input costs, to meet the economic needs of customers.

\section{Overall Design Scheme}

The controller uses Context-M3 core STM32F103 series of 32-bit microprocessor, which is widely used in industrial control, automotive electronics, medical equipment and other industries. STM32 has a wealth of internal resources as well as external expansion capabilities, the clock frequency can reach $72 \mathrm{MHZ}$, so it has a faster processing speed, can meet the requirements of high-speed communications[3]. According to the specific requirements of parking equipment, controller through real time detect the sensor signal, after a preset algorithm, the control signal is transmitted to the relay, then relay control motors. In the master-slave control system, the 
subordinative controller can exchange data though RS485 or CAN bus with the host controller [4].

The controller should have enough I/O ports to detect the sensor signals, STM32 has as many as 112 multifunctional bidirectional I/O port[5], all the I/O port can be mapped to the 16 interrupts, and almost all of the $\mathrm{I} / \mathrm{O}$ are able to tolerate $5 \mathrm{~V}$ signal, thus greatly improve the practicability and anti-interference of the controller[6]. Figure 1 is the three layers and six places parking equipment, it has six parking spaces, motors M1, M2, M3 provide electrical power output, the upright can be moved back and forth on the rail, and the mounter can move up and down along the uprights, also can rotate $90^{\circ}$ around the uprights, through sensors to restrict their movements. During operation, the controller can carry real-time voice prompts, if an accident occurs, Such as overloading of vehicles, equipment failure, and the vehicle exceed limited height, the corresponding sensor can sent the abnormal condition to the controller promptly, the controller stop the motors right now, and at the same time play voice prompts, to draw attention to safety.

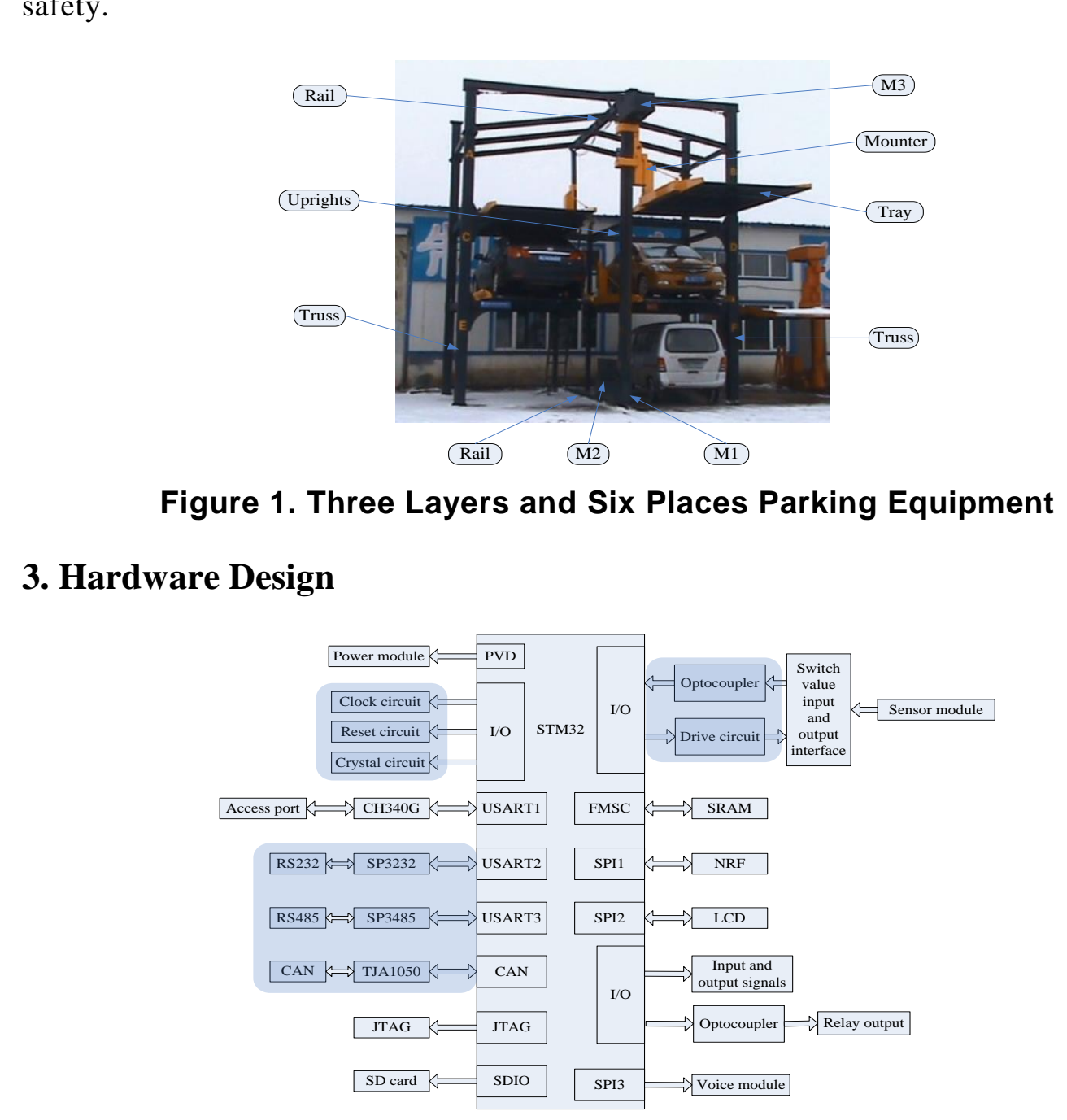

Figure 2. Hardware Structure of the Controller 
The hardware of the controller uses the modular design, which can not only guarantee the independence of each module, and also can realize the connection of various parts of the mutual [7]. Make full use of the STM32 on-chip rich peripherals, according to the required control functional of the multi-layer parking equipment, to design of communication module, power module, relay module and voice module, the hardware structure of the controller is shown in Figure 2.

\subsection{Power Module}

The design of power module is directly determine the stability of the controller, during the power module design should pay full consideration to the isolation, noise reduction, protection and other aspects. According to the need of parking equipment, power module must be able to provide the standard $3.3 \mathrm{~V}, 5 \mathrm{~V}, 12 \mathrm{~V}$ voltage. So we use the LM2576-12 to reduce the external switching power supply voltage of $24 \mathrm{~V}$ down to $12 \mathrm{~V}$ for relays. Toensure the power supply stability and anti-jamming, we select DC-DC isolated poyer modules B1205S-2W to reduce $12 \mathrm{~V}$ to $\mathrm{VDD}(+5 \mathrm{~V})$ for the use of sensors and optocouplers in addition the voltage stabilizer of LM2576-5 can output standard $+5 \mathrm{~V}$ powe for the use of ADuM1201,CH340G and other chips which need +5 power. Then though the linear regulator LM1117-3.3 buck to 3.3V supply to the STM32F103 and other chips which need $+3.3 \mathrm{~V}$ power like SP3485. At the same time the two trace $+5 \mathrm{~V}$ power use different GND, so this can avoid the electromagnetic interference which the optocouplers and Holzer sensors bring to the control system, the power module schematic diagram shown in Figure 3.

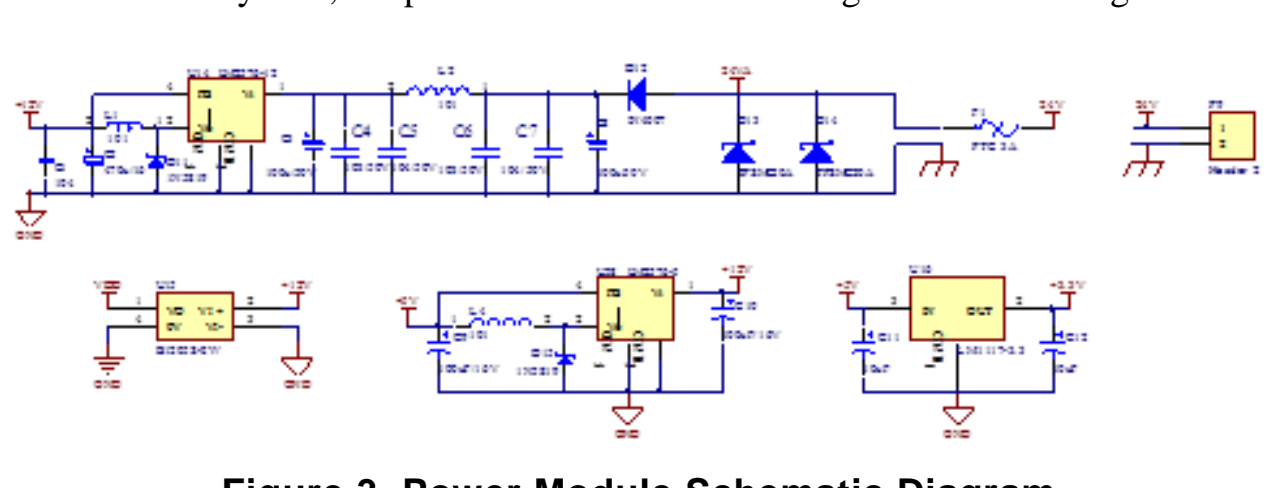

F1 in Figure 2 is a fuse, it can avoid short circuit and overload damage to the controller; Inductance L1, L2 are used to stop the AC interference signals through, to suppress the electromagnetic interference, but also can filter the power; Capacitors used to bypass, decoupling and filtering, D13 and D14 are TVS play the role of regulator.

\subsection{CAN Bus Communication Interface}

As STM32 integrated CAN2.0A / B controller, so just add a TJA1050 driver chips can be realize communication, in CAN bus interface circuit[8], the application of high speed optocoupler ADUM1201 to isolate the TJA1050 and STM32 chip, because if the bus speed is higher than $62.5 \mathrm{kpbs}$ is considered to the high speed mode. As shown in Figure 4, there is a 22PF parallel connection capacitance between CANH and GND, as well as CANL, used to filter out high frequency interference signals on the bus. In addition, between the two CAN bus access terminal and earth respectively, reverse connect a protection diode, when the CAN bus has a high negative voltage, by the diode circuit can play certain role of overvoltage protection. 


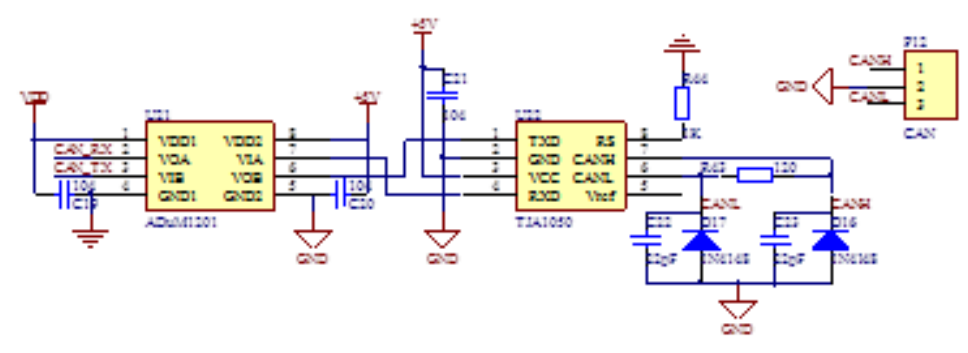

Figure 4. CAN Bus Communication Interface Circuit

\subsection{Switch Value Input and Relay Output Interface Circuit}

The sensors used in multi-layer parking equipment return signal most are the switching value, so it can directly use the STM32 general purpose I/O interface circuit connected to the sensor to get the test data. As shown in Figure 5, using TLP181 optocoupler to isolate. Pullup resistor prevent interference and the diode prevent current flow backward, the point of INT0 is used to detect whether there are data inputs in input port of INPUTO.

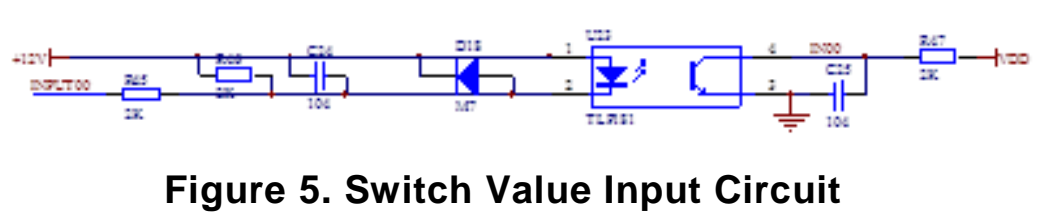

The multi-layer parking equipment putpot signats are generally relay output, as shown in Figure 6, we use a high voltage output with open eollector Hex Buffer/Driver SN74LS07. A SN74LS07 has six outputs, each channel can provide maximum output voltage of $30 \mathrm{~V}$, fully meet the relay coil control requinements. In addition, the relay coil with a parallel connection transient reverse diode to release reverse discharge current, protection relays and pre-driver chip, the point of INT0 is used to detect whether the relay is closed.

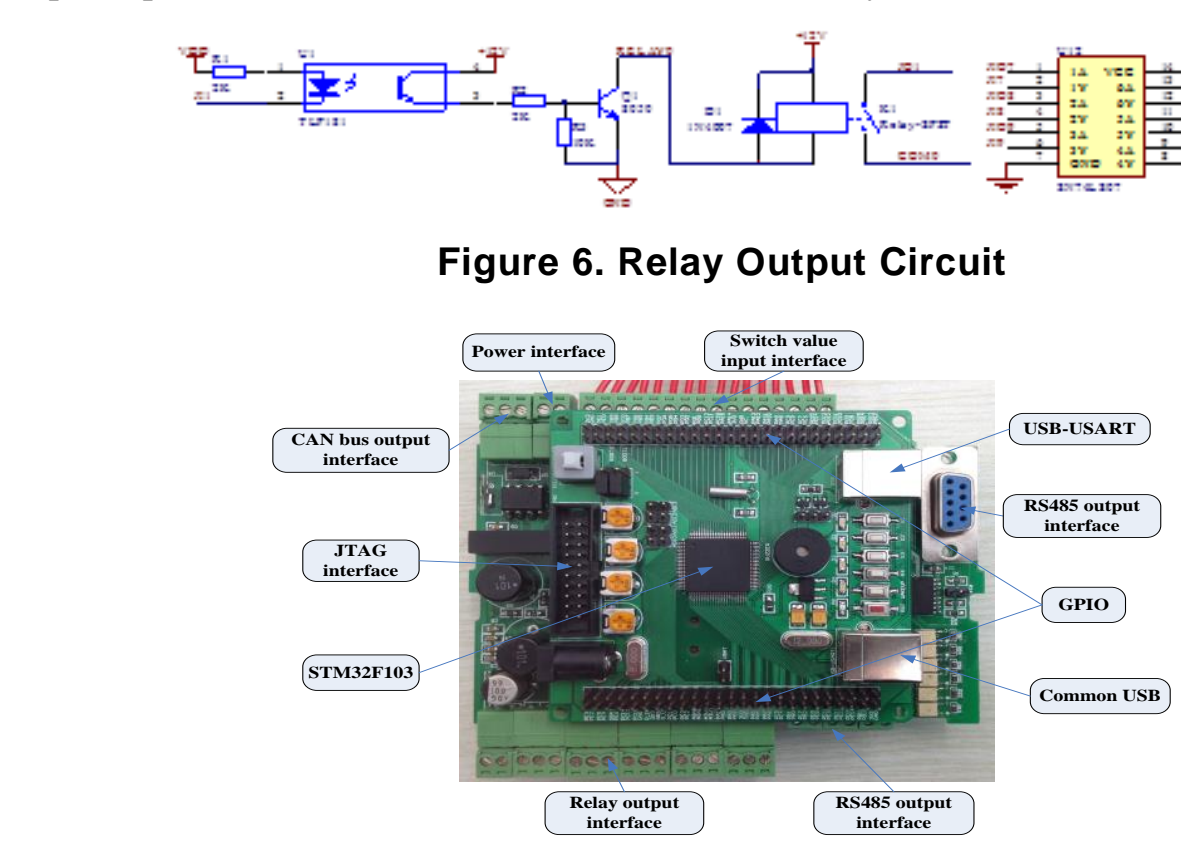

Figure 7. The Controller Circuit Board 
Figure 7 is the controller circuit board. In order to facilitate the connection, the controller is divided into two layers, in which the upper layer are STM32F103 minimum system and peripheral circuit, oscillator circuit, reset circuit, USB serial port, The upper plate can also be used as a simple controller; The lower is the peripheral interfaces, such as CAN bus communication interface, input interface, relay output interface.

\section{Software Architecture Design and Application Verification}

Many parking equipments are over reliance on hardware and neglect the software design, the software structure design based on good hardware resources can reflects the system's userfriendly [9]. Based on the control need of the three layers and six place parking equipment, design the software control flow. Control mode is divided into automatic and manual operation, and manual operation is apply to manual adjustment when the system can not fun automatically due to equipment malfunctions or other problems. The automatic mode-is the normal way of the multi-layer parking equipment, and can automatically park and withdraw the vehicle according to the customer demand, Figure 8 shows the main progran flow chart of the parking system with three layers and six parking equipment. The controller makes a judgment of parking or taking the car according to user input, then enter the corresponding subroutine.

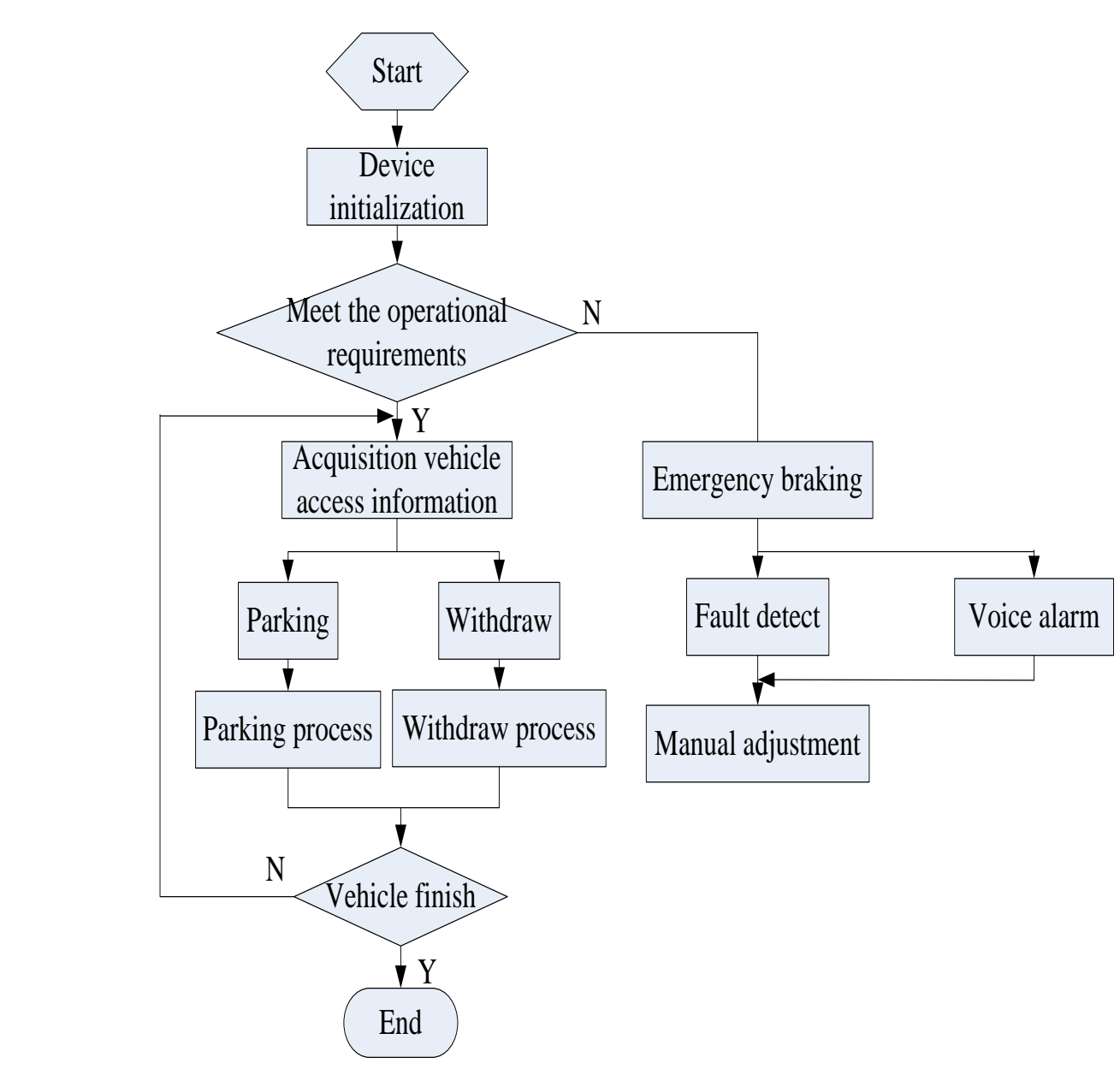

Figure 8. Main Program Flow Chart 
The parking process is basically the same as pick up. Here only introduce the parking process, the control system based on the information of user input to determine the parking, then find free place, according to predetermined algorithm processes to finish the parking process, the parking process diagram as shown in Figure 9.

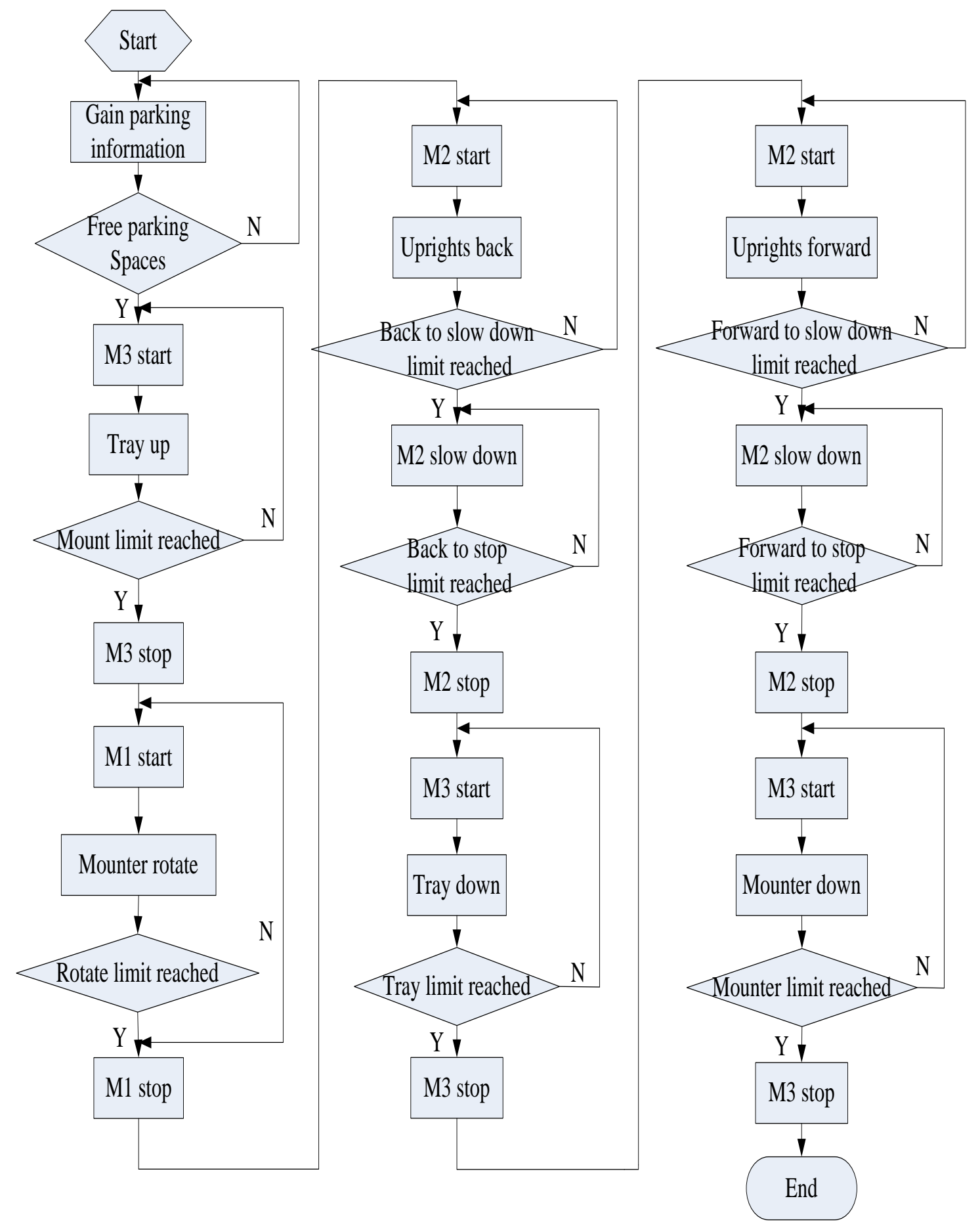

Figure 9. Parking Process Diagram 


\section{Conclusion}

Multi-layer parking equipment can make full use of the space resource, and the controller is the most important. The controller we design based on STM32 can increase integration, at the same time can reduce the control volume, decrease costs and easy maintenance. During the hardware design, we considered the isolation, filtering and other factors. After the schematic and PCB drawing, we proceed some necessary simulation, at the same time with high processing speed and the communication rate, stable power supply, can satisfy the control demand. Finally, we use the controller to control the three layers and six parking equipment, in combination with the equipment working process, we write the control software, over all aspects of the test, the controller's input and output is very stable, faster communication speed, each module can run better, can fully meet the requirements of the parking equipment control.

\section{Acknowledgements}

This research was supported by Heilongjiang Province Research and Development of Applied Technology Project (Grant No.GC13A410).

\section{References}

[1] Y. Li, J. Huang and J. Huang, "Research on Intelligent basket type parking equipment control system", vol. 50, no. 572, (2012), pp. 34-36.

[2] E. Fang, X. Chen and J. Zhou, "Control Part Design of Up-Down and Loft-Right”, vol. 19, no. 2, (2006), pp. 13-14.

[3] T. Pan, X. Ye, N. Zhang and J. Fan, "Desi gn and Application of CAN Bus Repeater Based on STM32", vol. 1, (2011), pp. 46-48.

[4] ST Company.ST A013 ST A013B STA013T mpeg2 5 layer III audio decoder, (2004).

[5] F. Yang, H. Ge and J. Zhang, "Design and implementation of embedded experimental system based on STM32 processor", vol. 42, no. 3, (2013), pp. 57-61.

[6] B. Deng, "Design and inplementation of elevator caller controller based on STM32", University of Electronic Science and Technology of China, (2011).

[7] L. Zhu, Y. Guo and Q. Li "Research and implementation of universal industrial controller based on STM32", vol. 2, no. 39, (2011), pp. 224-227

[8] T. Chanhun Park D. Il Park, J.-H. Kyung, K. Park and D. Kim, "Design of Dual Arm Robot Manipulator Using Modular Actuators with CAN Communication Networks”, COEX, Seoul, Korea, (2008) October 1417.

[9] S. Cheng, "Design and implementation of vehicle access controller based on STM32", vol. 4, (2012), pp. 2024.

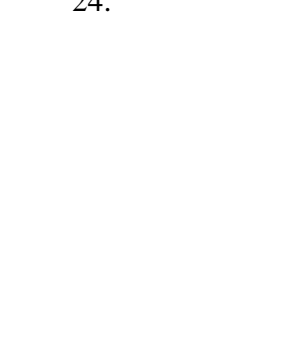


International Journal of Smart Home

Vol.8, No.1 (2014)

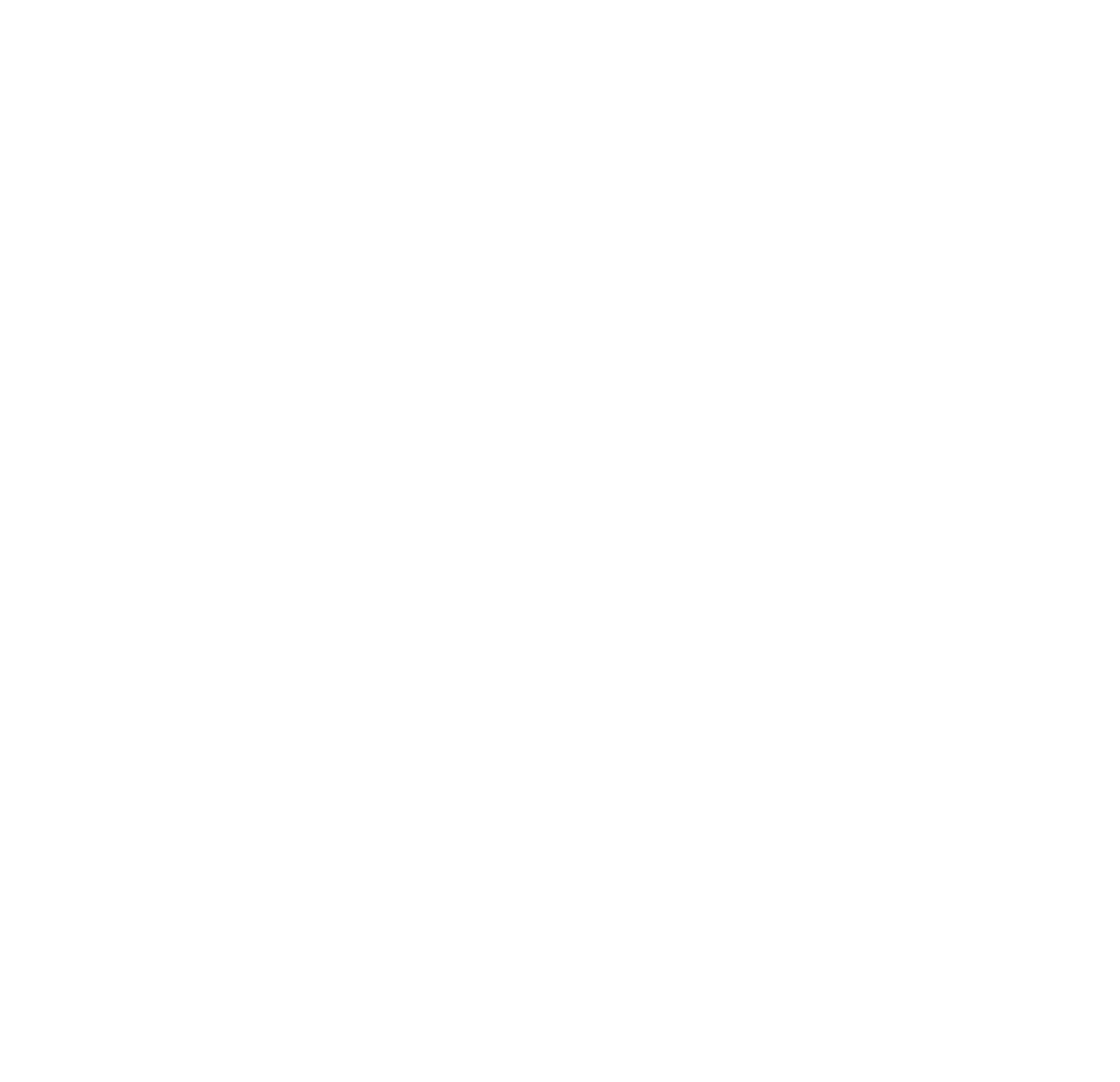

